\title{
Numerical solution of fuzzy differential equations by multistep block method
}

\begin{abstract}
Modeling of certain complex problems in science and engineering involve fuzzy differential equations. Many fuzzy differential equations cannot be solved analytically because of the complexity of the modeled problems. In this paper, a multistep block method is proposed for numerically solving first order fuzzy differential equations using Hukuhara differentiability. The convergence of the proposed method has been shown and the numerical results are presented.
\end{abstract}

Keyword: Fuzzy differential equation; Multistep block method 